\title{
Improving communication about cancer screening: moving towards informed decision making
}

\author{
Jolyn K Herscha,c, Brooke L Nickel ${ }^{a}$, Alex Ghanouni ${ }^{b}$, Jesse Jansen and \\ Kirsten J McCaffery ${ }^{a}$
}

a Sydney School of Public Health, University of Sydney, NSW, Australia

${ }^{b}$ Research Department of Behavioural Science and Health, University College London, UK

c Corresponding author: jolyn.hersch@sydney.edu.au

\section{Article history}

Publication date: July 2017

Citation: Hersch JK, Nickel BL, Ghanouni A, Jansen J, McCaffery KJ. Improving communication about cancer screening: moving towards informed decision making. Public Health Res Pract. 2017;27(3):e2731728. https://doi. org/10.17061/phrp2731728

\section{Key points}

- Community views about cancer screening are very positive, which may be partly due to persuasive public communications

- Contemporary approaches are shifting towards the goal of enabling people to make informed decisions about their participation in screening

- Communication tools and methods such as decision aids and shared decision making can support people to make informed decisions about participating in screening

\section{Abstract}

For decades, public communications about cancer screening have used persuasive techniques with the aim of maximising the number of people being screened. However, perspectives have changed more recently to acknowledge that screening can lead to harm as well as benefit, and that it is important for service users to consider both. For some types of cancer screening, there is professional contention about whether benefits clearly outweigh harms. In light of this, an emerging trend in cancer screening communication is to try to support informed decision making - that is, to help people understand both the advantages and disadvantages of screening, allowing them to make individual decisions about their screening participation that reflect their informed preferences. In this review, we provide an overview of key theoretical and practical aspects of improving communication and supporting informed decision making about cancer screening, highlight relevant research and discuss future implications.

\section{Introduction}

Cancer screening involves a complex array of potential consequences, including benefits (e.g. reduced disease burden, mortality and morbidity; improved disease outcomes) and harms (e.g. false positives, false negatives, overdiagnosis, other physical and psychological harms). ${ }^{1}$ There is variation across cancer types and screening modalities in the possible consequences for the individual screened, the probabilities of the various outcomes and the ratio of benefits to harms. These variations underpin differences in recommendations about whether and how screening should be offered and implemented. Some forms of screening are generally regarded as advantageous (e.g. bowel cancer screening with the faecal occult blood test); some are viewed more negatively (e.g. prostate cancer screening with prostate-specific antigen testing); and others are seen to have a finer balance between benefits and harms (e.g. breast cancer screening with mammography). ${ }^{2}$ Heterogeneity of study methodologies, 
population characteristics and screening delivery can lead to conflicting results and uncertainty about estimates of benefits and harms ${ }^{2}$, which complicate the task of making policy decisions about whether and how screening should occur.

Despite these complexities, policy makers are increasingly seeking to enable informed decisions by providing balanced information on harms and benefits to potential screening participants. ${ }^{1}$ In this review, we give an overview of current research and practice relating to improving communication about cancer screening, including relevant examples across a range of contexts. At times, we focus on breast cancer screening as a case where communication issues have received intense attention in recent years.

\section{Public perceptions of screening}

Attitudes about cancer screening are highly positive among the general public, and screening is often supported uncritically. ${ }^{3-5}$ Qualitative research suggests that key reasons motivating people to undergo screening include taking responsibility for one's own health, feeling a social obligation to do so, and gaining reassurance. ${ }^{3} \mathrm{~A}$ landmark US survey documented widespread public enthusiasm for routine cancer screening, with $87 \%$ of respondents considering it "almost always a good idea". ${ }^{4}$ Very similar results were reported a decade later in a large British survey. ${ }^{5}$ People's commitment to screening was strong enough that $58 \%$ of women in the US survey said they would overrule a physician who suggested having less frequent cervical screening, whereas $77 \%$ of men would continue prostate screening, and $74 \%$ of men and women would continue bowel screening even if their physician recommended against it. ${ }^{4}$ These attitudes may be based on unrealistic expectations: a systematic review of studies that quantitatively assessed patient or public expectations of medical interventions, including 15 studies on cancer screening, found that participants tended to overestimate benefits and underestimate harms. ${ }^{6}$

\section{Conventional approach to providing information}

Public health messaging about cancer screening for many decades has largely reflected the positive views held by public health organisations, professional associations, patient advocacy groups, academics and clinicians. ${ }^{7}$ According to these favourable perspectives on screening, authorities are justified in using persuasive communication tools with the goal of maximising uptake. These messages can take diverse forms. One frequently used approach is to induce feelings of fear and vulnerability, then offer hope by framing screening as a simple method of protection. ${ }^{7}$ A common feature of many conventional approaches to screening communication is to emphasise benefits and minimise harms. ${ }^{2,7,8}$ For example, several international reviews (most recently by Gummersbach et al. ${ }^{9}$ ) have found that breast screening information materials overestimate benefits and underplay harms. Such communications have created highly positive community views of screening that engender normative expectations that screening is the 'right' thing to do. ${ }^{3}$

\section{Need for informed (shared) decision making}

Messaging about screening has been criticised in recent years as simplistic and overly positive..$^{2,7}$ It is argued that, rather than encouraging uptake, screening communication should aim to inform people about harms and benefits so that they can make informed decisions about participation. ${ }^{2,7}$ The ethical justification is that screening puts large numbers of healthy people at risk of possible harm by offering interventions they might not otherwise seek out. ${ }^{1}$ Advocates of this perspective note that screening can trigger a cascade of serious interventions with lasting consequences, and individuals who choose to screen (or not) must live with their decision and its repercussions. Individuals may weigh attributes differently depending on their personal circumstances and psychological characteristics - and some might reasonably decline screening. ${ }^{2}$ According to this perspective, screening decisions are a good example of the value of helping people be engaged and involved in decision making.

The terms 'informed decision making' and 'shared decision making' relate to public and patient involvement in healthcare decision making. Both terms encompass a process that enables a person to participate in making a healthcare decision, having learnt about the intervention and its likely consequences, and having considered their preferences. Shared decision making is conceptualised specifically in terms of patient-clinician consultations in which both parties express preferences and participate in making decisions. Shared decision making may therefore be more relevant to screening tests based in primary care (e.g. prostate cancer screening ${ }^{10}$ than those provided outside a clinical encounter (e.g. breast and bowel cancer screening), where informed decision making might be the more appropriate term.

\section{Supporting informed (shared) decision making with decision aids}

Decision aids are designed to support people's decision making about healthcare interventions in circumstances where more than one reasonable option is available, with benefits and harms that people value differently (i.e. where personal preferences are relevant). ${ }^{11}$ Decision aids make the decision explicit (e.g. to be screened or not), describe why the choice exists, provide information about options and their outcomes, and are intended to help 
people consider the options from their own perspective.

Randomised controlled trials in a variety of settings demonstrate that decision aids improve knowledge of risk and accuracy of risk perceptions, stimulate people to take a more active decision making role, and can improve congruence between personal values and the chosen option, usually without lengthening consultations. ${ }^{11}$ Decision aids can also assist informed decision making about screening participation. ${ }^{12}$

Uncertainties about the level of benefit and harm can make it difficult to determine what specific information to provide in decision support materials about cancer screening. Selecting the most appropriate data involves considering both the methodological quality of the evidence and its relevance to the local context. For transparency and to reduce bias, decision aids (or supplementary documents that describe their development) should cite the studies selected. ${ }^{13}$ When presenting quantitative information (e.g. frequencies of benefits and harms), risk communication research supports using either percentages (e.g. $x \%$ ) or frequency formats (e.g. $x$ in 1000) and specifying the denominator. ${ }^{14}$ Pictographs or icon arrays (e.g. as used by Smith et al. ${ }^{15}$ and Hersch et al. ${ }^{16}$ ) improve comprehension, including among people with low literacy and numeracy. ${ }^{14}$ Given that there is rarely any single 'right' number for a specific outcome probability, decision aids should also try to communicate the scientific uncertainty in some way (e.g. as described by Forbes and Ramirez $\left.{ }^{17}\right) .^{13}$

There are some concerns that encouraging laypeople to take a more active role in healthcare decisions might exacerbate inequalities. To examine this, a systematic review evaluated the impact of shared decision making interventions on health inequalities, including nine studies about cancer screening. ${ }^{18}$ The review concluded that such interventions significantly improve outcomes for disadvantaged groups $^{18}$, which suggests that a shared decision making approach for cancer screening is likely to be broadly beneficial, including for vulnerable populations. For example, an Australian trial of a bowel cancer screening decision aid for adults with low education ${ }^{15}$ achieved an $85 \%$ consent rate in a socio-economically disadvantaged population, with $93 \%$ of those randomised completing the trial. The decision aid increased the proportion of people making an informed choice by 22\% (as per the study definition $)^{15}$, providing evidence that informed decision making can be accessible to the wider community.

\section{Alternative approaches}

Although structured presentation of health information may empower people to assume a more active decision making role, some people may not wish to personally evaluate detailed screening information. ${ }^{17}$ Furthermore, despite considerable research evidence supporting decision aids ${ }^{11}$, integrating shared decision making into routine clinical practice continues to be a challenge. ${ }^{19}$ Alternatives include the 'consider an offer' approach, which aims to avoid overburdening people with unwanted information and tasks while respecting their autonomy ${ }^{8}$, and has been the basis for the approach to cancer screening communication in England since 2013. ${ }^{17}$ The model involves helping people evaluate the trustworthiness and personal relevance of the screening offer, acknowledging that it might reasonably be declined, and making further information available for those who want it. People who are eligible for cancer screening receive a letter of invitation from the National Health Service (which may be seen as a recommendation in itself), and a leaflet (developed at arm's length from the screening program) that provides more information about benefits and harms. The leaflet aims to encourage people to assess the offer of screening, rather than simply encouraging screening, and to make it clear that declining the offer may be justified. ${ }^{17}$

Ultimately, whether individual-level deliberation is to be promoted for a given healthcare decision is a policy judgement. Such judgements can be usefully guided by evidence on the preferences of well-informed members of the public. Engaging a 'community jury' (or 'citizens' jury'), in which a representative sample of the target population deliberates together about benefits and harms, has been demonstrated to be a feasible way of eliciting an informed, considered community view on questions of screening policy and communication. For example, an Australian community jury about prostate cancer screening concluded that men should be able to make informed choices, identified the need for better information, and recommended supporting general practitioners to better inform patients. ${ }^{10}$ This method has also been applied in the context of breast cancer screening. ${ }^{17}$

\section{Communicating about overdiagnosis}

One particularly contentious issue in some cancer screening contexts is overdiagnosis (or overdetection). A prominent concern in prostate, thyroid and breast cancer (with evidence emerging about lung cancer), overdiagnosis occurs when tumours are detected that would not become clinically apparent or cause death if left undetected and untreated. There is currently no way to reliably determine whether a given cancer will progress or remain indolent, so overdiagnosis typically leads to overtreatment, even among screen-detected cases that are recognised as low risk (e.g. low-grade prostate cancer).

Much of the shift in perspective towards promoting informed choice over increased uptake has been due to increasing concern about overdiagnosis, especially in breast cancer screening..$^{17}$ Public awareness about overdiagnosis of slow-growing cancers is limited. ${ }^{10,17,20} \mathrm{An}$ online survey of people aged 50-69 in the US who had been invited to participate in cancer screening by their doctor found that less than $10 \%$ of patients had been informed about the risk of overdiagnosis. ${ }^{21}$ Similarly, in an Australian telephone survey, $10 \%$ of women and $18 \%$ 
of men who had been screened for breast and prostate cancer, respectively, said they were told about the risk of overdiagnosis. ${ }^{22}$

Communicating the concept of overdiagnosis is difficult, partly because it is undetectable on an individual level, which means that understanding it is not intuitive. A randomised controlled trial in Australia aimed to improve women's understanding about overdiagnosis in breast cancer screening and assessed the effects of informing them. ${ }^{16}$ The decision aid explaining overdiagnosis increased knowledge and enabled more women to make an informed choice (as per the study definition), compared with a control decision aid omitting this information. Being better informed about potential consequences also made women feel slightly less positive about breast cancer screening and somewhat reduced intentions to be screened. This suggests that some women, when better informed, might make different decisions about participation. ${ }^{16}$ Similarly, a brief explanation of overdiagnosis reduced intentions to undergo thyroid cancer screening among Korean women. ${ }^{20}$

\section{Communication in context}

Crucially, evidence based information such as that presented in a decision aid is just one of many factors shaping healthcare choices. Decisions are influenced by emotions, not only scientific information. People's sense of risk is often more about feelings than facts, which might limit the impact of new factual information. ${ }^{23}$ Breast cancer screening, in particular, has been highlighted as a decision context exemplifying the tension between powerful emotions and objective risk assessment. ${ }^{23}$ This tension makes it challenging to communicate potential harms of screening.

The source of information may shape how individuals interpret new information. Clinicians tend to be respected as credible sources of advice and can influence public perceptions of cancer screening. ${ }^{24}$ If future information materials encourage more deliberative decision making, clinicians might be called on to help people understand the evidence and guide them in clarifying their preferences. Current evidence suggests that many clinicians perform suboptimally in explaining the downside of screening to patients. ${ }^{25}$ Clinicians may not yet have the capacity or training to participate usefully in shared decision making about screening, and messages currently conveyed by clinicians may be inconsistent with recently developed screening information. ${ }^{17}$ An important future goal should therefore be to develop ways to support clinicians in communicating more effectively with patients about cancer screening, and its benefits and harms, including overdiagnosis and other challenging topics. ${ }^{10}$

\section{Conclusion}

When communicating with the public about cancer screening, promoting uptake by emphasising benefits was once considered justified. However, for some types of cancer screening, there is professional contention about whether the benefits of screening clearly outweigh the harms. Arguments have been made that individuals should be supported to make informed decisions about whether to undergo screening, and that they need accessible, highquality information about screening outcomes to do so.

Despite challenges, research has shown that it is possible to help people become better informed about screening..$^{10,12,16}$ Based on recent evidence ${ }^{16,20}$, it appears that most people would still prefer to be screened, even when given new information about risks such as overdiagnosis. Engaging and supporting individuals to construct and enact their own informed preferences in making decisions about their healthcare is vital if patients and citizens are really to be at the heart of the healthcare system.

\section{Acknowledgements}

$\mathrm{JH}$ and $\mathrm{JJ}$ are supported by a National Health and Medical Research Council (NHMRC) Fellowship. AG's and KM's institutions received funding from Cancer Research UK and an NHMRC Program Grant, respectively, for their research in cancer screening.

\section{Competing interests}

None declared

\section{Author contributions}

$\mathrm{JH}$ was responsible for the design, drafting and editing of the manuscript. BN, AG, JJ and KM were responsible for reviewing and editing the manuscript, and contributed to the design of the manuscript.

\section{References}

1. Standing Committee on Screening of the Community Care and Population Health Principal Committee of the Australian Health Ministers' Advisory Council. Population based screening framework. Canberra: Commonwealth of Australia; 2016 [cited 2017 Jan 11]. Available from: www. cancerscreening.gov.au/internet/screening/publishing. nsf/Content/16AE0B0524753EE9CA257CEE0000B5D7/ \$File/Final\%20Population\%20Based\%20Screening\%20 Framework\%202016.pdf

2. Stefanek M. Uninformed compliance or informed choice? A needed shift in our approach to cancer screening. J Natl Cancer Inst. 2011;103(24):1821-6.

3. Willis K, Baxter J. Trusting technology: women aged 40-49 years participating in screening for breast cancer - an exploratory study. Aust N Z J Public Health. 2003;27(3):282-6. 
4. Schwartz LM, Woloshin S, Fowler FJ, Jr., Welch HG. Enthusiasm for cancer screening in the United States. JAMA. 2004;291(1):71-8.

5. Waller J, Osborne K, Wardle J. Enthusiasm for cancer screening in Great Britain: a general population survey. Br J Cancer. 2015;112(3):562-6.

6. Hoffmann T, Del Mar C. Patients' expectations of the benefits and harms of treatments, screening, and tests: a systematic review. JAMA Intern Med. 2015;175(2):274-86.

7. Woloshin S, Schwartz LM, Black WC, Kramer BS. Cancer screening campaigns - getting past uninformative persuasion. N Engl J Med. 2012;367(18):1677-9.

8. Entwistle VA, Carter SM, Trevena L, Flitcroft K, Irwig L, McCaffery K, Salkeld G. Communicating about screening. BMJ. 2008;337:a1591.

9. Gummersbach E, Piccoliori G, Oriol Zerbe C, Altiner A, Othman C, Rose C, Abholz HH. Are women getting relevant information about mammography screening for an informed consent: a critical appraisal of information brochures used for screening invitation in Germany, Italy, Spain and France. Eur J Public Health. 2010;20(4):409-14.

10. Rychetnik L, Doust J, Thomas R, Gardiner R, Mackenzie G, Glasziou P. A community jury on PSA screening: what do well-informed men want the government to do about prostate cancer screening - a qualitative analysis. BMJ Open. 2014;4(4):e004682.

11. Stacey D, Legare F, Lewis K, Barry MJ, Bennett CL, Eden $\mathrm{KB}$, et al. Decision aids for people facing health treatment or screening decisions. Cochrane Database Syst Rev. 2017;4:CD001431.

12. van Agt HM, Korfage IJ, Essink-Bot ML. Interventions to enhance informed choices among invitees of screening programmes - a systematic review. Eur J Public Health. 2014;24(5):789-801.

13. Joseph-Williams N, Newcombe R, Politi M, Durand M-A, Sivell S, Stacey D, et al. Toward minimum standards for certifying patient decision aids: a modified Delphi consensus process. Med Decis Making. 2014;34(6):699-710.

14. Trevena LJ, Zikmund-Fisher BJ, Edwards A, Gaissmaier W, Galesic M, Han PK, et al. Presenting quantitative information about decision outcomes: a risk communication primer for patient decision aid developers. BMC Med Inform Decis Mak. 2013;13:S7.
15. Smith SK, Trevena L, Simpson JM, Barratt A, Nutbeam D, McCaffery KJ. A decision aid to support informed choices about bowel cancer screening among adults with low education: randomised controlled trial. BMJ. 2010;341:c5370.

16. Hersch J, Barratt A, Jansen J, Irwig L, McGeechan K, Jacklyn $G$, et al. Use of a decision aid including information on overdetection to support informed choice about breast cancer screening: a randomised controlled trial. Lancet. 2015;385(9978):1642-52.

17. Forbes LJ, Ramirez AJ, Expert group on Information about Breast Screening. Offering informed choice about breast screening. J Med Screen. 2014;21(4):194-200.

18. Durand MA, Carpenter L, Dolan H, Bravo P, Mann M, Bunn F, Elwyn G. Do interventions designed to support shared decision-making reduce health inequalities? A systematic review and meta-analysis. PLoS ONE. 2014;9(4):e94670.

19. Joseph-Williams N, Lloyd A, Edwards A, Stobbart L, Tomson D, Macphail S, et al. Implementing shared decision making in the NHS: lessons from the MAGIC programme. BMJ. 2017;357:j1744.

20. Lee S, Lee YY, Yoon HJ, Choi E, Suh M, Park B, et al. Responses to overdiagnosis in thyroid cancer screening among Korean women. Cancer Res Treat. 2016;48(3):883-91.

21. Wegwarth $O$, Gigerenzer $G$. Less is more: overdiagnosis and overtreatment: evaluation of what physicians tell their patients about screening harms. JAMA Intern Med. 2013;173(22):2086-7.

22. Moynihan R, Nickel B, Hersch J, Beller E, Doust J, Compton S, et al. Public opinions about overdiagnosis: a national community survey. PLoS ONE. 2015;10(5):e0125165

23. Rosenbaum L. Invisible risks, emotional choices - mammography and medical decision making. N Engl J Med. 2014;371(16):1549-52.

24. Ahn HS, Welch HG. South Korea's thyroid-cancer "epidemic" - turning the tide. N Engl J Med. 2015;373(24):2389-90.

25. Hoffman RM, Elmore JG, Fairfield KM, Gerstein BS, Levin CA, Pignone MP. Lack of shared decision making in cancer screening discussions: results from a national survey. Am J Prev Med. 2014;47(3):251-9.

\section{Copyright: (c) (7) (2) ()}

(C) 2017 Hersch et al. This article is licensed under the Creative Commons Attribution-NonCommercial-ShareAlike 4.0 International Licence, which allows others to redistribute, adapt and share this work non-commercially provided they attribute the work and any adapted version of it is distributed under the same Creative Commons licence terms. See: www.creativecommons.org/licenses/by-nc-sa/4.0/ 